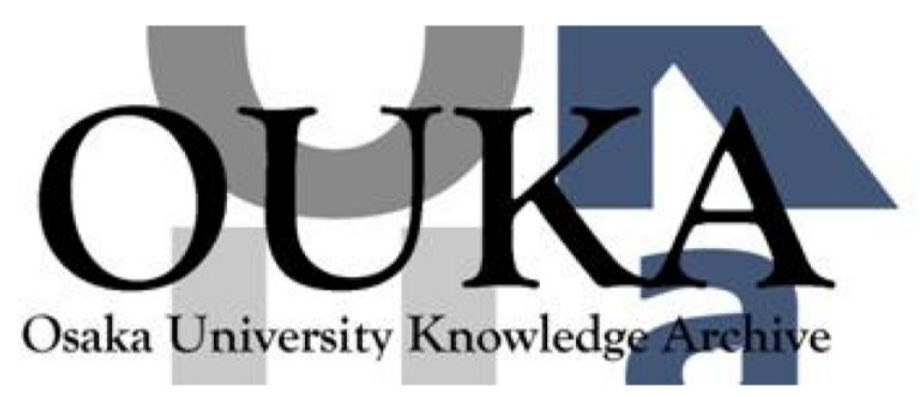

\begin{tabular}{|c|c|}
\hline Title & $\begin{array}{l}\text { A relation between membrane permeability and } \\
\text { flow rate at low Reynolds number in circular } \\
\text { pipe }\end{array}$ \\
\hline Author (s) & $\begin{array}{l}\text { Takeuchi, Shintaro; Tazaki, Asahi; Miyauchi, } \\
\text { Suguru et al. }\end{array}$ \\
\hline Citation & Journal of Membrane Science. 582 p. 91-p. 102 \\
\hline Issue Date & $2019-07-15$ \\
\hline oaire:version & AM \\
\hline URL & https://hdl. handle. net/11094/79018 \\
\hline rights & $\begin{array}{l}\text { - } 2019 \text { Elsevier Ltd. This manuscript version is } \\
\text { made avai lable under the Creative Commons } \\
\text { Attribution-NonCommercial-NoDerivatives } 4.0 \\
\text { International license. }\end{array}$ \\
\hline Note & \\
\hline
\end{tabular}

Osaka University Knowledge Archive : OUKA

https://ir. Library. osaka-u. ac. jp/

Osaka University 


\title{
A relation between membrane permeability and flow rate at low Reynolds number in circular pipe
}

\section{Shintaro Takeuchi ${ }^{1 *}$, Asahi Tazaki ${ }^{1}$, Suguru Miyauchi ${ }^{2,3}$ and Takeo Kajishima ${ }^{1}$}

\author{
${ }^{1}$ Dept. of Mechanical Engineering, Osaka University, 2-1 Yamada-oka, Suita-city, Osaka 565-0871 Japan \\ ${ }^{2}$ Institute of Fluid Science, Tohoku University, 2-1-1 Katahira, Aoba-ku, Sendai, Miyagi, 980-8577, Japan \\ ${ }^{3}$ Dept. of Mechanical Engineering, University College London, Torrington Place, London, WC1E 7JE, UK \\ * Corresponding author: shintaro.takeuchi@mech.eng.osaka-u.ac.jp
}

\section{Abstract}

A relation between membrane permeability to a pure solvent (i.e., inverse of resistance to a flow through membrane) and flow rate is derived in a circular pipe driven by a constant pressure difference across the pipe length. Membrane is assumed to be non-deformable and zero thickness, and the permeate flux of pure solvent due to pressure discontinuity across the flat membrane is coupled with the governing equations for incompressible Newtonian fluid in the Stokes regime. The permeation flow rate (normalised by that of the Hagen-Poiseuille flow for the no-membrane case or with a membrane of infinite permeability) is represented as a function of a non-dimensional permeability including the aspect ratio of the pipe geometry. The relation is established through comparison with a fully-validated numerical simulation result: the numerical discretisation is based on our original discrete-forcing immersed boundary method, which guarantees (i) conservations of mass and momentum even in the immediate vicinity of the membrane surface and (ii) consistency between incompressible velocity and pressure fields. Inverse analysis of the above formula yields the permeability as function of the flow rate through the pipe, comprising three equations covering the entire permeability ranges (from low to high permeabilities). The established permeability formulae are expected to be useful for identifying effective permeabilities of membrane to single-component fluid or pure solvent in practical applications.

Keywords: Permeable membrane; Permeability; Pressure discontinuity; Discrete-forcing immersed boundary method 


\section{Introduction}

Permeable membranes play an important role in various fields such as separation/purification/selection process and living/artificial biological environments. Typical examples are chemical separation [1], desalination of sea water by reverse osmosis membrane [2, 3], oxygen transport by red blood cells [4] and drug delivery assessment in blood vessels [5], intestines [6] and/or lung [7, 8, 9, 10]. Estimating permeability $L_{p}$ (to a pure solvent) is of importance for assessing membrane performance and efficiency. In recent advancement in nano-scale filtration, Zhou et al. [11] showed that, by applying an electric field, water permeation through graphene-oxide membrane (millimetres in diameter) can be controlled in either of the two distinct states: ultrafast-permeation and complete-blockage states. Nevertheless, for industrial and biological applications, desire of passive-control of membrane is still strong through modulations of membrane structure (i.e., pore size distribution, membrane thickness, surface area, etc) and surface properties for adopting to mechanical and/or chemical environment ( $\mathrm{pH}$, ionic-strength, etc).

In ultra-filtration (for separating macro-molecular solute from the solvent by externally-applied pressure difference $\Delta p$ ), for example, the permeate flux deviates from the pure solvent flux in low- $\Delta p$ range, whereas, by increasing $\Delta p$, the permeate flux becomes less sensitive to the induced pressure difference [12]. The osmotic pressure model [12] explains that these trends are determined by the balance between the pure-solvent permeation and osmosis by solute transport, and permeability $L_{p}$ can be calculated from both processes given a set of parameters. For the former case, local permeate flux of pure solvent, $J_{v}$, is linearly correlated with the pressure discontinuity across membrane, $[p]$, with the coefficient $L_{p}$, and this relation may be used for direct determination of $L_{p}$ (and the $L_{p}$ calculation from osmosis is mentioned later). However, local permeate flux deviates from a uniform distribution in a cross-section, particularly near the solid boundary (e.g., pipe wall) where membrane is supported or fixed, and therefore, the pressure distribution is not uniform over the cross-section, either. Even if a mean flux over the cross-section is accurately measured, the error in $\llbracket p \rrbracket$ underestimates $L_{p}$.

Permeability of a membrane of layered-structure, such as graphene oxide, can be modelled with several parameters of geometric configuration, and the predicted permeability on the continuum scale shows good agreement with a molecular-dynamics simulation result [13]. However, for general porous membrane of non-layered structure, a micro-scopic model of permeability $L_{p}$ is based on a Hagen-Poiseuille flow through a micro pore of circular cross-section across membrane thickness [14]:

$$
L_{p}=\frac{r_{p}^{2}}{8 \mu} \frac{A_{r}}{\vartheta}
$$


where $\mu$ is the viscous coefficient, $r_{p}$ the equivalent pore radius penetrating the membrane with mean tortuosity being unity, $\vartheta$ the membrane thickness and $A_{r}$ the correction coefficient representing the effective opening ratio of pores for the solvent flow. In the above estimate, two parameters $r_{p}$ and $A_{r} / \vartheta$ characterise the property of membrane permeability. Representative estimation methods of pore size include microscopic measurement $[15,16]$, mercury porosimetry method $[17,18]$, bubble-point method $[19,20]$ and thermoporometry method [21]. Interestingly, Nakao and Kimura [22] estimated $A_{r} / \vartheta$ by solute permeation with a given $L_{p}$ in their theoretical study of solute transport, and found that $A_{r} / \vartheta$ values for different Stokes radii of the solute deviate from the estimated value from the pure-water permeation (Eq.(1)). Conversely, the estimated $L_{p}$ based on the micro-scopic quantities involves statistic dispersion. The above points suggest that both direct measurement of $L_{p}$ and indirect estimation could be contaminated with statistic uncertainty, which would influence the prediction and design of micro- and ultra-filtration processes. This justifies the necessity of accurate estimation method of $L_{p}$.

In this study, we present theoretical relations between membrane permeability $L_{p}$ and flow rate $Q$ of an incompressible Newtonian fluid, $Q=F\left(L_{p}\right)$, in a non-dimensional form and its inverse $L_{p}=F^{-1}(Q)$. The flow impeded by a non-deformable flat membrane in a circular pipe under a constant external pressure difference $\Delta p$ is studied in the Stokes regime [23], where the effect of convection is negligible in comparison to the viscous effect. Note that our formula is based on macro-scopic mass conservation and represented by flow rate (a macro-scopic value) in a pipe, which would attain a reduced level of statistical dispersion from the micro-scopic scale, and the formula explicitly includes the effect of supporting wall as the boundary condition. Inverse formula is constructed for three characteristic ranges of permeability by piecewise lowdimensional functions based on expansions with polynomial and rational functions [24, 25], and the errors in the respective ranges are addressed.

To confirm the effectiveness, the $Q-L_{p}$ formula is compared with a numerical result using a fullyvalidated numerical method for solving the permeation flow through a membrane with keeping the consistency of the incompressible velocity and pressure fields. In general, for resolving the permeation flow through pores of membrane, many grid points of smaller scale than pore are necessary. However, if the effect of membrane is given as a boundary condition at a continuum scale, the feasible spatial scale largely extends. Therefore, in the present study, a membrane of zero-thickness is incorporated into the discretised Navier-Stokes and continuity equations. For numerical simulation of mass transfer across membrane, the present authors have pointed out that, in order to reproduce the permeation of incompressible fluid through 


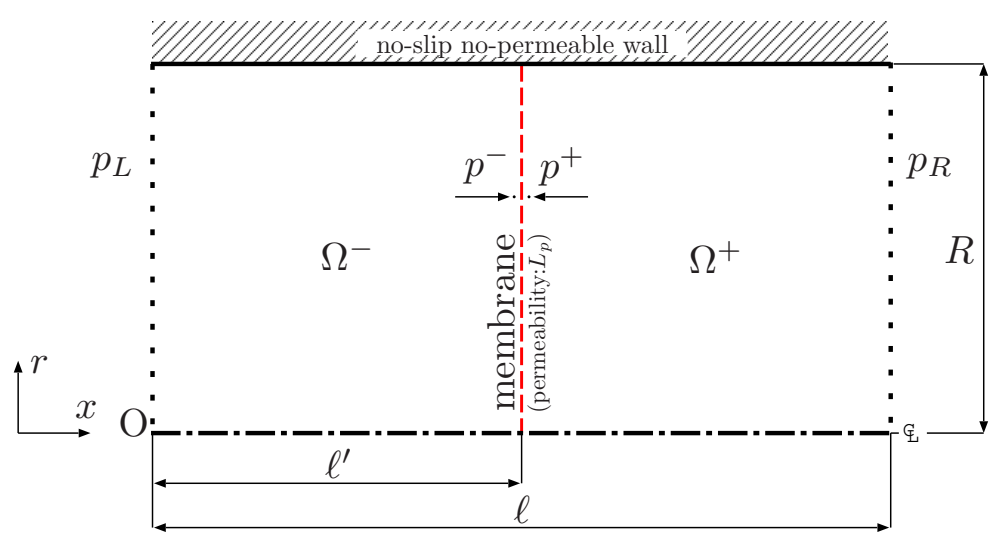

Figure 1: Schematic of the computational domain of circular pipe.

a membrane on a fixed fluid mesh system (generally non-conforming to the membrane surface), sharp representation of the membrane, especially capturing the trans-membrane pressure difference $\llbracket p \rrbracket$, is of critical importance [26]. Our discrete-forcing immersed boundary method [27, 28] on a fixed Cartesian mesh is suitable for this purpose, as pressure distribution corresponding to the no-slip and no-permeable conditions is obtained by solving the mass and momentum conservations even in the immediate vicinity of the membrane (without determining the velocity near the interface by interpolation). In the present study, a permeate flux is incorporated into the discretisation of the governing equations and a new pressure Poisson equation is derived to allow pressure discontinuity across permeable membrane in a consistent manner with the incompressible velocity field. Through comparison with the numerical result by the above method, the $Q-L_{p}$ formula is established.

\section{Flow rate through a membrane in a circular pipe}

The flow rate of pressure-driven flow through a circular pipe impeded by a membrane is derived. The fluid domain is set up as Fig. 1, and the flow is driven by a constant pressure difference, $p_{L}-p_{R}(\equiv \Delta p)$, across the distance $\ell$ in the longitudinal $(x)$ direction. The membrane is placed at $x=\ell^{\prime}$ in parallel with the $r$ axis (i.e., perpendicular to the centreline). Deflection of the membrane is not taken into account.

In case of no membrane, fully-developed velocity profile is the axisymmetric Hagen-Poiseuille flow:

$$
u_{\mathrm{P}}(r)=-\frac{\alpha}{4 \mu}\left(R^{2}-r^{2}\right)
$$

where $r$ is the radial distance from the centre axis, $R$ the pipe radius, $\mu$ the viscous coefficient and $\alpha$ the prescribed pressure gradient, $-\Delta p / \ell$. Hereafter, the flow rate of the Hagen-Poiseuille flow is denoted as $Q_{\mathrm{P}}\left(=-\alpha \pi R^{4} / 8 \mu\right)$. 
By placing a permeable membrane, the above parabolic velocity profile is no longer valid. However, with a membrane of large permeability $L_{p}$, we can reasonably expect that the parabolic profile is asymptotically recovered. Therefore, in the following, we assume a large-permeability case, and an approximate velocity profile is obtained.

To reflect the effect of a permeable membrane on the local velocity profile, $\alpha$ in Eq.(2) is temporarily replaced with $\mathrm{d} p / \mathrm{d} x$, and the equation is integrated with respect to $x$ in the ranges $0 \leq x \leq \ell^{\prime}$ and $\ell^{\prime} \leq x \leq \ell$ separately:

$$
\begin{aligned}
\ell^{\prime} u_{L} & =-\frac{1}{4 \mu}\left(p^{-}-p_{L}\right)\left(R^{2}-r^{2}\right), \\
\left(\ell-\ell^{\prime}\right) u_{R} & =-\frac{1}{4 \mu}\left(p_{R}-p^{+}\right)\left(R^{2}-r^{2}\right),
\end{aligned}
$$

where $u_{L}$ and $u_{R}$ are, respectively, the fluid velocities in the left- and right-hand sides of the membrane, and $p^{\mp}$ are the limiting pressure values on the interface as Fig. 1 shows. Those velocities are equated with the on-membrane velocity $\widetilde{u}(r)$, and $\ell^{\prime}$ is eliminated from the above equations:

$$
\ell \widetilde{u}=-\frac{p_{R}-p_{L}+p^{-}-p^{+}}{4 \mu}\left(R^{2}-r^{2}\right) .
$$

Then, the pressure jump $\left(p^{-}-p^{+} \equiv[p]\right)$ is substituted with the volumetric permeate flux multiplied by the resistance $\left(L_{p}^{-1}\right)$ :

$$
\llbracket p \rrbracket=L_{p}^{-1} \widetilde{u}
$$

and also, substituting $p_{R}-p_{L}$ back with $\alpha \ell$, we obtain the velocity restricted by the membrane as:

$$
\widetilde{u}=-\frac{\alpha \ell}{4 \mu} \frac{R^{2}-r^{2}}{\ell+\left(4 \mu L_{p}\right)^{-1}\left(R^{2}-r^{2}\right)} .
$$

In the asymptotic situation of $L_{p} \rightarrow \infty$, Eq.(4) coincides with Eq.(2).

The flow rate $Q\left(=\int_{0}^{R} 2 \pi r \widetilde{u} \mathrm{~d} r\right)$ normalised by $Q_{\mathrm{P}}$ is represented as

$$
\frac{Q}{Q_{\mathrm{P}}}=8 M\left(1+4 M \log \left[\frac{4 M}{1+4 M}\right]\right),
$$

where $M$ is non-dimensional permeability including the geometric parameter of the domain (i.e., pseudo aspect ratio of the pipe length divided by the pipe radius):

$$
M=\frac{L_{p} \mu}{R} \cdot \frac{\ell}{R}
$$

Note that $M$ is essentially the same form as $A_{r}$ in Eq.(1), although the spatial scales are different. Hereafter, the relation of Eq.(5) is denoted as $Q=F(M)$ for simplicity. 


\section{Outline of the numerical method}

In this section, a numerical method for solving permeation flow is briefly explained based on our original discretisation approach for discrete forcing (DF) immersed boundary (IB) method, which offers a sharp interface treatment by incorporating the boundary conditions for the interfacial velocity into the discretised governing equations.

The present authors developed a method that directly discretises the N-S equation even at the grid points adjacent to the interface, while, at the same time, ensuring the consistency between the incompressible velocity and pressure fields $[27,28]$. By their "consistent direct discretisation" for the DF-IB approach, the non-slip condition on the interface was strictly imposed in a discrete sense while satisfying the mass and momentum conservations, which enables capturing the sharp distribution of the velocity and pressure at the interface.

The governing equations (Eqs.(A1) and (A2) in Appendix A.1) are discretised by a finite difference method. The flow variables are defined on the collocated arrangement, and the spatial discretisation is by second order central finite difference.

The convective and viscous terms in the vicinity of the interface (i.e., adjacent cell to the interface) are discretised by considering the distance from the cell centre to the membrane (Eqs.(A4) and (A5)), and those two terms are time-updated by the 4th-order Runge-Kutta method. A fractional step method is employed for coupling the velocity and pressure fields, and, the velocity is corrected given a pressure field at the next time step (Eq.(A7)). By substituting the corrected velocity field (Eq.(A7)) into the discretised momentum conservation equation, a pressure equation taking account of the distance to the interface (Eq.(A6) for nopermeable case) is derived in a consistent form to the discretised velocity field. For discretising on the cells not adjacent to the interface, ordinary discretisation of 2nd-order accuracy is adopted. The method has been fully validated for stationary solid case [27] and moving/deforming membrane case [28].

For the present study, to incorporate permeate flux across membrane, the above pressure equation (Eq.(A6)) is modified to the equations for the respective sides of the membrane (Eqs.(A12) and (A13)) to allow the trans-membrane flux from one side to the other. Then, the velocity components in the righthand sides of those equations are replaced with the pressure jump $\llbracket p \rrbracket$ across membrane by using the linear permeate flux model (Eq.(A3)), and the equation for the permeated pressure field is obtained (Eqs. (A17) and (A18)). The pressure jump in the pressure equation is decomposed by extrapolation from the respective 
sides of the membrane, so that the final form of the pressure equation constitutes a closed system in terms of the pressure values in the surrounding cells.

The details of the numerical method are found in Appendix A.2 along with verifications with analytical prediction and independently-conducted numerical result by a monolithic strong coupling method [26].

\section{Results and discussion}

Permeation flow through a membrane in a circular pipe driven by a constant pressure difference is discussed. The predicted flow rate in Eq.(5) is validated through comparison with the numerical result by a discreteforcing immersed boundary method [27, 28] considering permeation across membrane. Further, inverse formulae representing the permeability with the flow rate are discussed.

\subsection{Velocity profile and flow rate}

For the configuration in Fig. 1, numerical simulation is conducted under $\Delta p=1$, $\ell=5 R$ and $\ell^{\prime}=$ $\ell / 2$. The grid spacing $\Delta$ is uniform in both directions, and the spatial resolution is $R / \Delta=40$, unless specified otherwise. The Reynolds number based on the maximum velocity (i.e., the centreline velocity) for no-membrane case and the pipe radius is set to unity. The numerical method in $\S 3$ is modified to an axisymmetric coordinate system for the present problem. The fluid variables (velocities and pressure) are defined on the collocated arrangement (i.e., on the cell centres of the rectangular cells), and the membrane is aligned with the cell faces along the $r$ axis, although any membrane configurations with respect to the cell centres are available as Fig. A2(b) in Appendix A.3 shows.

Figure 2 compares the velocity profiles obtained by the numerical simulation and analytical prediction $\widetilde{u}$. The following six $M$ values are selected for simulating the flow field: $M=0.005,0.05,0.5,5,50$ and 500 . The symbols in the figure are the velocity profiles on the membrane obtained by the numerical simulation and the line represents $\widetilde{u}$. 


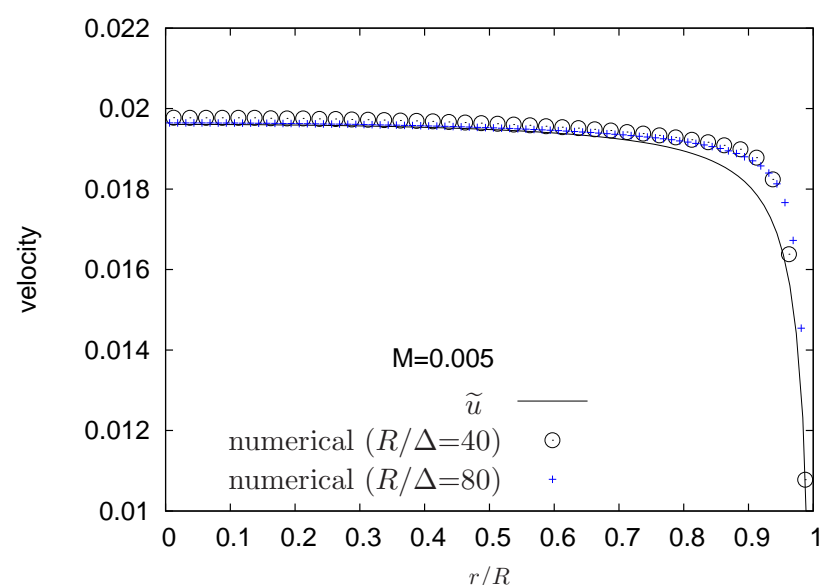

(a) $M=0.005$

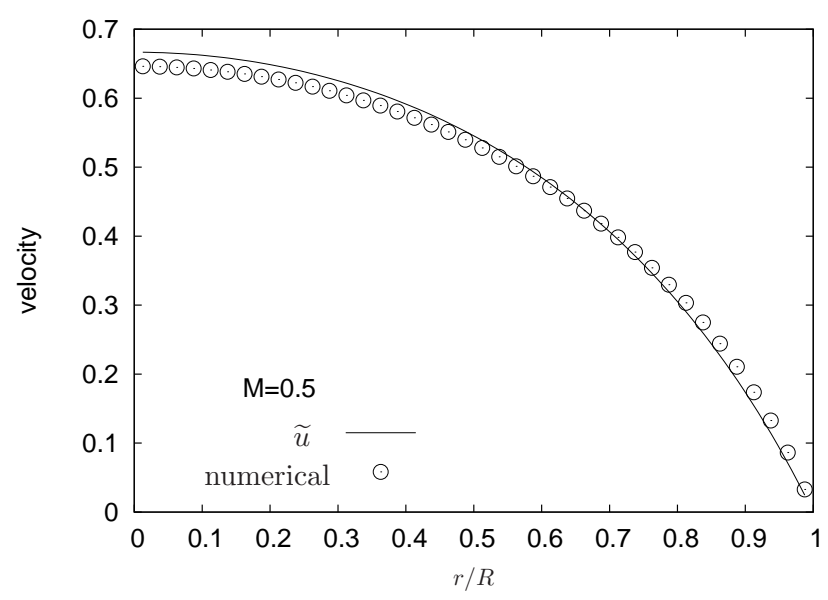

(c) $M=0.5$

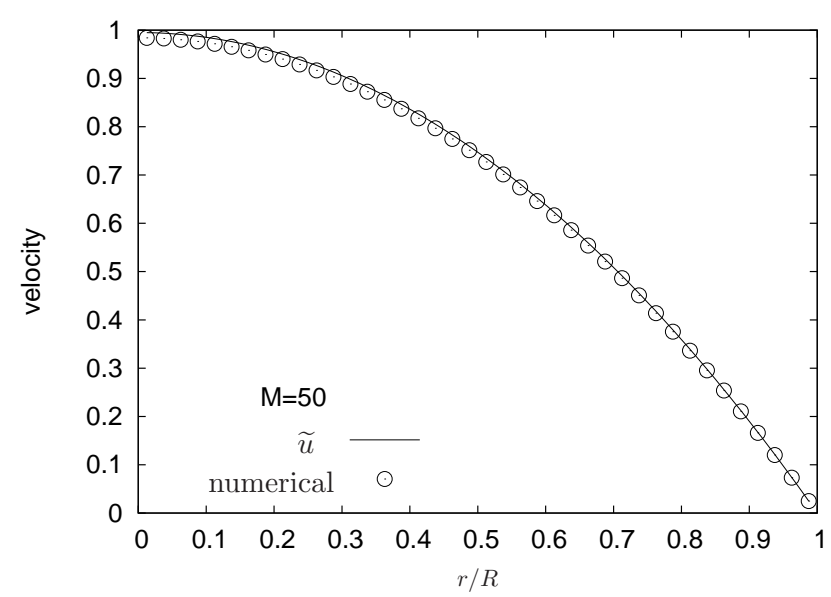

(e) $M=50$

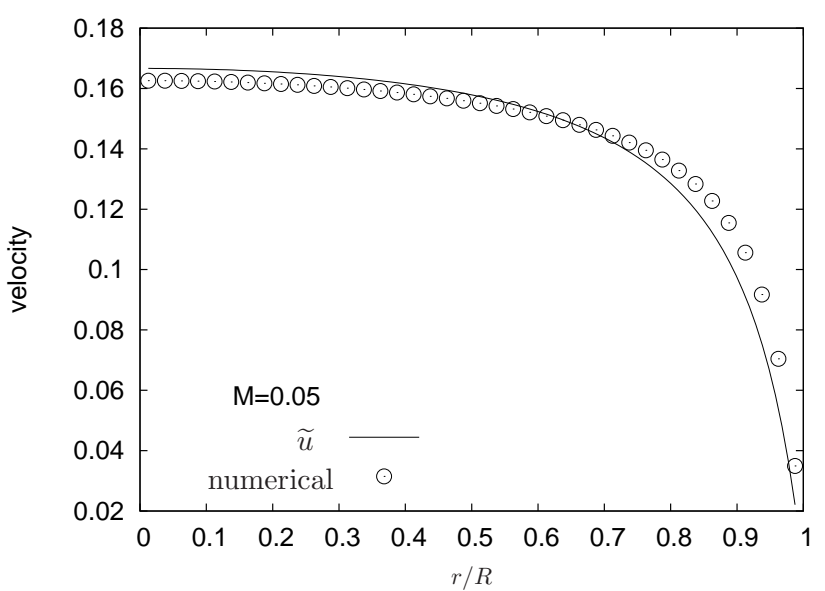

(b) $M=0.05$

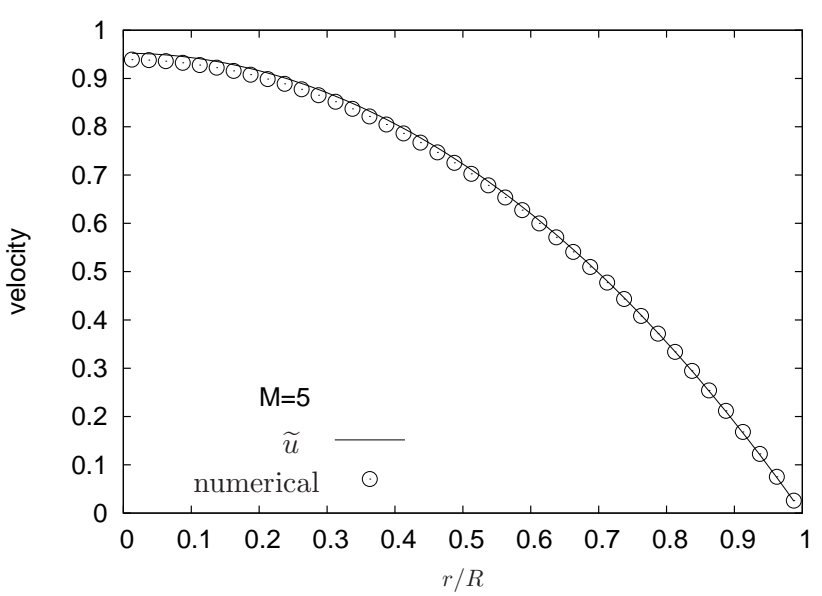

(d) $M=5$

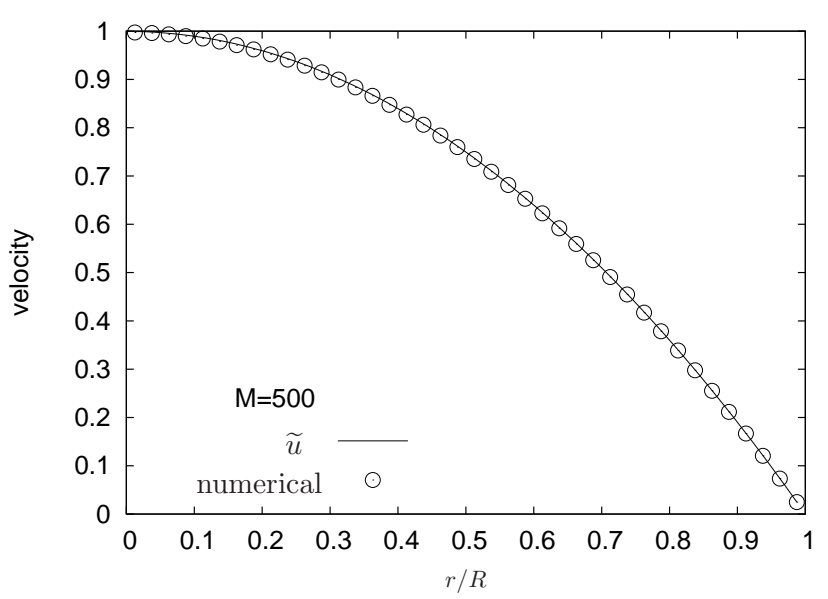

(f) $M=500$

Figure 2: Velocity profiles on the membrane obtained by the present numerical method (symbols) for the following six values of the non-dimensional permeability: $M=0.005,0.05,0.5,5,50$ and 500 . The numerical result is compared with the theoretical profile, Eq.(4), normalised by the maximum velocity (viz. centreline velocity) of the no-membrane case ( $M=0$, i.e., Hagen-Poiseuille flow), represented by line. The spatial resolution of the numerical results is $R / \Delta=40$, except for the case (a) $M=0.005$ which also compares with the result of $R / \Delta=80$. 


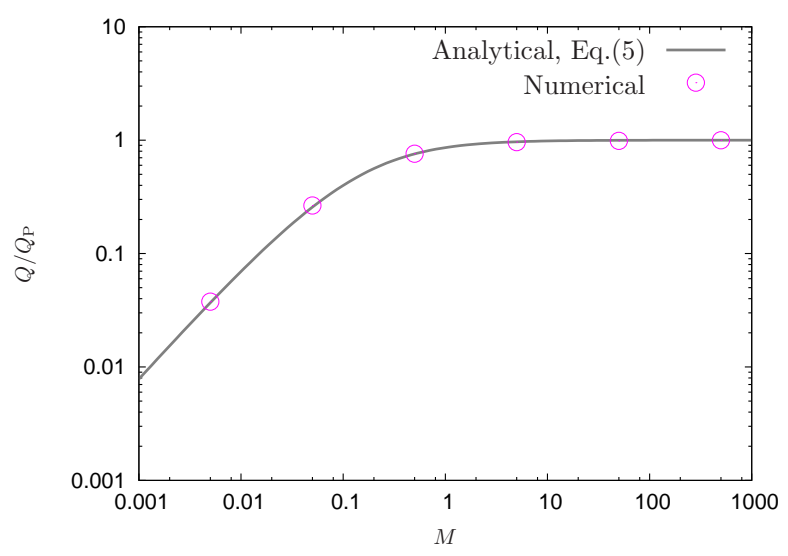

Figure 3: Normalised flow rate $Q / Q_{\mathrm{P}}$ as a function of non-dimensionalised permeability $M$. Analytical prediction Eq.(5) is compared with the numerical result by the method in $\S 3$ implemented in the axisymmetric coordinate system.

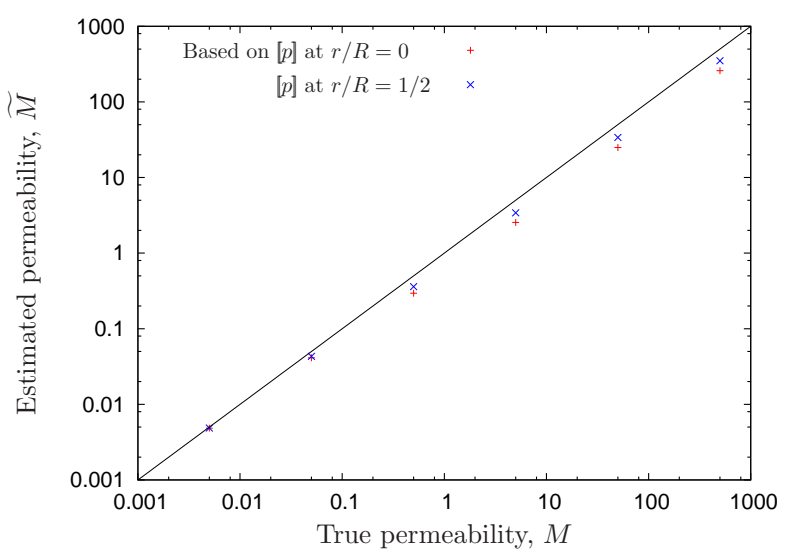

Figure 4: Estimated permeability $\widetilde{M}$ against true $M$. Permeability is estimated by dividing the mean crosssectional velocity $Q / \pi R^{2}$ with $[p \rrbracket$ obtained at two different radial sampling points of $r / R=0$ and $1 / 2$. The solid line shows $\widetilde{M}=M$.

The figure shows that, as $M$ value becomes small, the resistance by membrane is significant. Although, the predicted velocity profiles $\widetilde{u}$ do not fit the numerical result, $\widetilde{u}$ for $M \leq 0.5$ approximately captures the trend and the discrepancy is readily reduced by increasing the spatial resolution as Fig. 2(a) shows. As $M$ is increased, the model closely predicts the velocity profiles of the numerical result, as expected.

Despite the disagreement in the cross-sectional velocity profile in the low- $M$ range, the flow rate is expected to show good agreement as the numerical method tightly conserves mass and momentum and Eq.(5) also takes the mass conservation into account. Figure 3 summarises the normalised flow rates, $Q / Q_{\mathrm{P}}$, for the above six $M$ values. The graph shows that Eq.(5) reasonably predicts the flow rates of the pipe flow impeded by a membrane for different permeabilities over six orders of magnitude, suggesting the wide applicability of Eq.(5) irrespective of membrane micro-structure or fluid species.

To highlight the effect of radial variation of $\llbracket p \rrbracket$, estimation of permeability is attempted based on a $\llbracket p \rrbracket$ at a local sampling radial distance. Permeability $\widetilde{L_{p}}$ is calculated by dividing the cross-sectional mean velocity 
$\bar{u}=Q / \pi R^{2}$ with a locally-measured $[p]$ :

$$
\begin{aligned}
\widetilde{L_{p}} & =\frac{\bar{u}}{\llbracket p \rrbracket}=-\frac{\alpha R^{2}}{8 \mu \llbracket p \rrbracket} \frac{Q}{Q_{\mathrm{P}}} \\
\therefore \quad \widetilde{M} & =\frac{\widetilde{L_{p}} \mu}{R} \cdot \frac{\ell}{R}=\frac{1}{8} \frac{\Delta p}{\llbracket p \rrbracket} \frac{Q}{Q_{\mathrm{P}}} .
\end{aligned}
$$

Figure 4 plots the estimated permeability $\widetilde{M}$ against the true $M$. The pressure jump is obtained at two different radial sampling points $r / R=0$ and $1 / 2$ from the numerical result. The plots below the line $\widetilde{M}=M$ suggest that simply correlating the local pressure jump and the mean velocity in the pipe significantly underestimates the permeability.

In the next subsection, it is shown that inverse formula $M=F^{-1}(Q)$ can be constructed with less error compared to the above estimation (7), which would eliminate the necessity of measuring $\llbracket p \rrbracket$ across membrane in experiments for estimating $M$.

\subsection{Approximate functions for $Q$ and $M$}

If inverse function $M=F^{-1}(Q)$ is found, this has an advantage for identifying a permeability $M$ (or $L_{p}$ ) from a measured flow rate in a circular pipe, which may be useful for industrial applications. However, it is difficult to construct the inverse function of Eq.(5) in a form of single function that covers the entire $M$ range.

On the other hand, as Fig. 3 suggests, the $Q-M$ curve may have three distinct regions: a linear region ( $M \lesssim 0.1)$, a flat region $(M \gtrsim 10)$ and the intermediate (connecting) region.

In the following, inverse function is constructed for each region with piecewise lower-order functions and $(j, k)$-Padé approximant, which is a rational function of $j$-th and $k$-th order polynomials for numerator and denominator.

\section{(i) Linear region at low- $M$ range}

The following expansions are obtained for Eq.(5):

$$
\begin{aligned}
& \text { 1st-order : } \frac{Q}{Q_{\mathrm{P}}}=8 M \quad+O\left(M^{2}\right) \\
& \text { 2nd-order : } \frac{Q}{Q_{\mathrm{P}}}=8 M(1+4 M \log (4 M)) \quad+O\left(M^{3}\right)
\end{aligned}
$$

Figure 5(a) plots the above two equations, and Fig.5(b) shows the error from Eq.(5). Although the second-order expansion covers a wider range of $M$ than the first order expansion (Fig. 5(a)), 


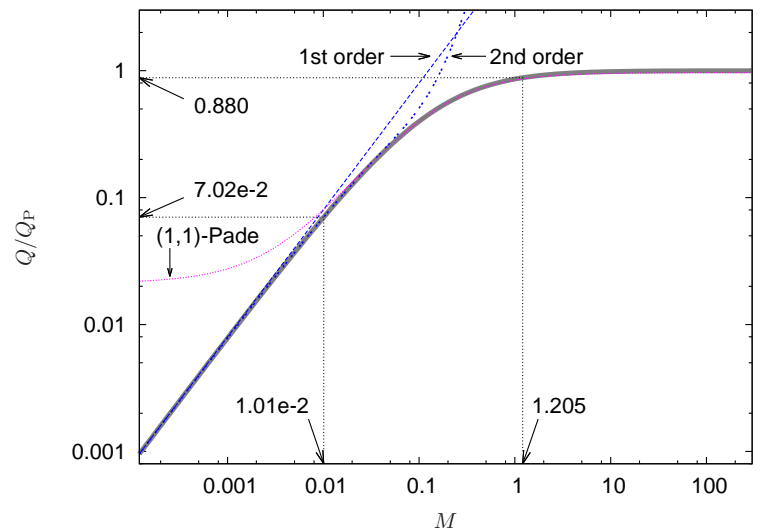

(a) Flow rate, Eqs.(8) and (9)

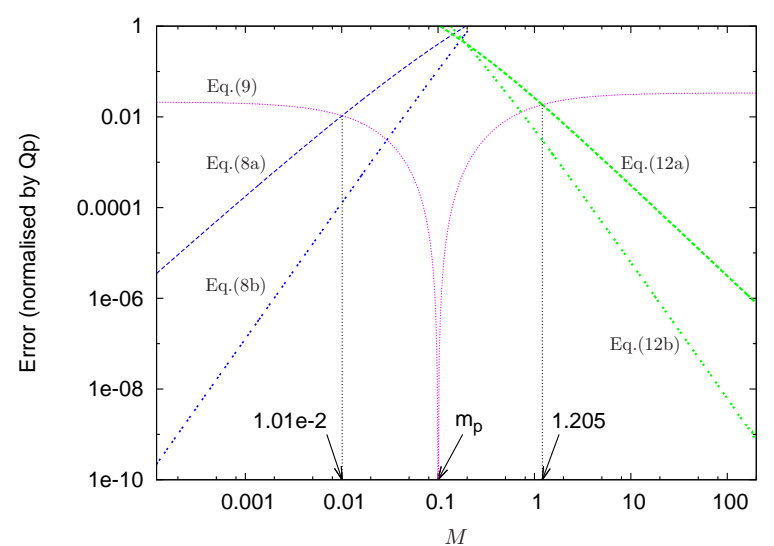

(b) Errors in Eqs.(8), (9) and (12)

Figure 5: (a) Approximate functions of $Q / Q_{\mathrm{P}}$, Eqs.(8)(9), for the low and intermediate $M$ ranges. (b) The errors in Eqs.(8), (9) and (12) for the entire $M$ range.

the transcendental equation (8b) cannot be inverted as $M=F^{-1}(Q)$. Any higher-order Taylor expansions than second order do not improve the approximation due to the log-dependent term, and it is even difficult to have inverse functions of those. On the other hand, although the linear approximation, Eq.(8a), restricts the applicable range, the simpler structure may be more useful than Eq.(8b) in terms of inversion.

\section{(ii) Intermediate region: $(1,1)$-Padé approximation}

For the intermediate region, we employ rational function for approximating $Q=F(M)$ around $M=m_{\mathrm{p}}$. Here, $(1,1)$-Padé approximation around $m_{\mathrm{p}}=10^{-1}$ gives the following result:

$$
\frac{Q}{Q_{\mathrm{P}}}=\frac{1}{25} \frac{50(1+620 M)+460(1-80 M) \log \frac{7}{2}+392(-1+30 M) \log ^{2} \frac{7}{2}}{205-475 M+49(-3+10 M) \log \frac{7}{2}} .
$$

The plot of Eq.(9) in Fig. 5 shows that the above equation is reasonably approximates Eq.(5). However, the error in Eq.(9) increases as $\left|M-m_{\mathrm{p}}\right|$ increases, taking the saturating values of $2.12 \times 10^{-2}(M \rightarrow 0)$ and $3.36 \times 10^{-2}(M \rightarrow \infty)$, as Fig.5(b) shows.

The above equation is solved with respect to $M$ in the following form:

$$
M=\frac{25 \frac{Q}{Q_{\mathrm{P}}}\left(-205+147 \log \frac{7}{2}\right)+50+460 \log \frac{7}{2}-392 \log ^{2} \frac{7}{2}}{125 \frac{Q}{Q_{\mathrm{P}}}\left(-95+98 \log \frac{7}{2}\right)-40\left(775-920 \log \frac{7}{2}+294 \log ^{2} \frac{7}{2}\right)} .
$$

In general, $(k, k)$-Padé approximant gives better approximation of Eq.(5) as $k$ increases. For example, the saturating error trends are summarised in Tab.1. Moreover, those Padé approximants are invertible as far as the $k$ range in the table. However, the form is complex and may not be very practical. Therefore, we do not explore further higher-order approximations here. 
Table 1: Asymptotic errors in $(k, k)$-Padé approximant around $m_{\mathrm{p}}=10^{-1}$ with respect to Eq.(5)

\begin{tabular}{c|c|c}
$k$ & $\mid$ Error $\mid(M \rightarrow 0)$ & $\mid$ Error $\mid(M \rightarrow \infty)$ \\
\hline 1 & $2.12 \times 10^{-2}$ & $3.36 \times 10^{-2}$ \\
2 & $4.05 \times 10^{-3}$ & $2.66 \times 10^{-3}$ \\
3 & $1.27 \times 10^{-3}$ & $2.28 \times 10^{-4}$ \\
4 & $5.18 \times 10^{-4}$ & $2.01 \times 10^{-5}$ \\
5 & $2.50 \times 10^{-4}$ & $2.00 \times 10^{-6}$
\end{tabular}

On the other hand, (1,1)-Padé approximant of Eq.(5) around an arbitrary $m_{\mathrm{p}}$ would be useful as it gives the exact $Q$ value at $M=m_{\mathrm{p}}$ (see Fig. 5(b)) and, therefore, approximation in the intermediate region can be improved around $M=m_{\mathrm{p}}$ at a desired level by adjusting $m_{\mathrm{p}}$. Here, a general form of Eq.(9) is derived for an arbitrary $m_{\mathrm{p}}$ and it is solved with respect to $M$. In the following, only the result is presented for reference:

$$
M=m_{\mathrm{p}} \frac{G_{2} Y^{2}+G_{1} Y+G_{0}}{H_{2} Y^{2}+H_{1} Y+H_{0}},
$$

where

$$
\begin{aligned}
Y & =4 m_{\mathrm{p}} \log \left(\frac{4 m_{\mathrm{p}}}{1+4 m_{\mathrm{p}}}\right) \\
G_{2} & =8\left(4 m_{\mathrm{p}}+1\right)^{2}, \\
G_{1} & =16 m_{\mathrm{p}}\left(16 m_{\mathrm{p}}+3\right)+\frac{3\left(4 m_{\mathrm{p}}+1\right)^{2}}{m_{\mathrm{p}}} \frac{Q}{Q_{\mathrm{P}}}, \\
G_{0} & =16 m_{\mathrm{p}}\left(8 m_{\mathrm{p}}-1\right)+\frac{48 m_{\mathrm{p}}^{2}+18 m_{\mathrm{p}}+1}{m_{\mathrm{p}}} \frac{Q}{Q_{\mathrm{P}}}, \\
H_{2} & =24\left(4 m_{\mathrm{p}}+1\right)^{2}, \\
H_{1} & =8\left(6 m_{\mathrm{p}}+1\right)\left(16 m_{\mathrm{p}}+3\right)+\frac{\left(4 m_{\mathrm{p}}+1\right)^{2}}{m_{\mathrm{p}}} \frac{Q}{Q_{\mathrm{P}}}, \\
H_{0} & =8\left(48 m_{\mathrm{p}}^{2}+10 m_{\mathrm{p}}+1\right)+2\left(8 m_{\mathrm{p}}+3\right) \frac{Q}{Q_{\mathrm{P}}} .
\end{aligned}
$$

\section{(iii) Flat region at high- $M$ range}

At higher- $M$ range, the Padé approximation still fits the curve (Fig.5(a)), but it is not necessarily the best approximation as the saturating trend of the error shows in Fig. 5(b). On the other hand, Eq.(5) shows $Q / Q_{\mathrm{P}} \rightarrow 1$ as $M \rightarrow \infty$. Therefore, in the high- $M$ region, Eq.(5) is expanded with respect to $M^{-1}$ around $M^{-1}=+0$ :

$$
\begin{array}{ll}
M^{-1} \text {-order }: \frac{Q}{Q_{\mathrm{P}}}=1-\frac{1}{6 M} & +O\left(M^{-2}\right), \\
M^{-2} \text {-order }: \frac{Q}{Q_{\mathrm{P}}}=1-\frac{1}{6 M}+\frac{1}{32 M^{2}} & +O\left(M^{-3}\right) .
\end{array}
$$


The above equations give better approximation as Fig. 5(b) shows that the error decreases as $M$ increases.

In summary, inverse function in each region is suggested as follows:

$$
M=\left\{\begin{array}{lr}
\frac{1}{8} \frac{Q}{Q_{\mathrm{P}}} & (0.0101>M) \\
\text { Eq. }(10) & (0.0101 \leq M \leq 1.205) \\
\frac{1}{6}\left(1-\frac{Q}{Q_{\mathrm{P}}}\right)^{-1} & (M>1.205)
\end{array}\right.
$$

and the corresponding boundary $Q / Q_{\mathrm{P}}$ values for the respective regions are shown in Fig. 5(a).

Note that, when using Eq.(11), the applicable $M$ range in Eq.(13) must be adjusted. The error levels of the above piecewise low-order inverse functions are sufficiently low (Fig.5(b)) in comparison to those in Fig. 4. The results suggest the significance of the formulae (5) and (13).

\section{Conclusion}

A formula relating the flow rate $Q$ in a circular pipe and membrane permeability $L_{p}$ (to a pure solvent) is established by comparing with numerical result with a fully-validated numerical solver, and its inverse functions are constructed with piecewise low-order functions, thereby enabling estimation of membrane permeability from a measured flow rate in the Stokes regime without information of trans-membrane pressure discontinuity.

In the numerical study, a permeate flux model was incorporated into the strategy of "consistent direct discretisation", and a new pressure Poisson equation was derived. Sharp pressure discontinuity over one computational cell is facilitated by the two strategies: direct-discretisation strategy of the governing equations in the immediate vicinity of the interface and the consistent-coupling strategy between the incompressible velocity and pressure fields at the permeable interface. The validity of the numerical method was established through comparison with independently-conducted numerical result by a strong monolithic coupling method for both permeable and non-permeable conditions.

Because any specific materials or pore structure are not assumed for membrane in the present study, the established formula and inverse functions relating effective permeability and flow rate through it are universal as long as permeate flux is proportional to the pressure discontinuity and the flows in both sides of the membrane are in the Stokes regime. 


\section{Appendix A Method}

\section{A.1 Governing equations}

For numerical method of permeation flow in the next subsection, the governing equations are summarised here.

Membrane is represented by Lagrangian marker points, whereas the fluid motion is solved in an Eulerian frame on a Cartesian fixed mesh non-conforming to the membrane geometry.

Throughout the present study, membrane is stationary.

The fluid is an incompressible Newtonian fluid with constant density $(\rho)$ and constant viscosity $(\mu)$. The governing equations of the fluid are the equation of continuity and Navier-Stokes (N-S) equation:

$$
\begin{aligned}
& \nabla \cdot \boldsymbol{u}=0 \\
& \frac{\partial \boldsymbol{u}}{\partial t}+\boldsymbol{u} \cdot \nabla \boldsymbol{u}=-\nabla p+\frac{1}{\operatorname{Re}} \nabla \cdot\left[(\nabla \boldsymbol{u})^{T}+(\nabla \boldsymbol{u})\right]
\end{aligned}
$$

where $\boldsymbol{u}$ is the velocity, $t$ the time, $p$ the pressure, Re the Reynolds number based on the reference velocity $U$ and reference length $H$. The pressure is non-denationalised by $\rho U^{2}$.

Volumetric flux across membrane is modelled by the following equation:

$$
\boldsymbol{J}_{v}=\operatorname{Re} L \llbracket p \rrbracket \boldsymbol{n},
$$

where $\boldsymbol{n}$ is the unit normal vector pointing from the rear-side of the membrane $\Omega^{-}$to the front-side $\Omega^{+}$, $L$ the non-dimensionalised permeable coefficient defined with the membrane permeability $L_{p}$ as $L_{p} \mu / H$ and $\llbracket p \rrbracket$ the hydraulic pressure jump (or pressure discontinuity) across the membrane given with the limiting pressure values on the interface in the respective sides of the membrane, $p^{-}$and $p^{+}$, as $\llbracket p \rrbracket=p^{-}-p^{+}$.

\section{A.2 Computational method}

In this subsection, a numerical formulation of permeation flow is explained based on our numerical method, and the method is validated by comparing with both analytical and independently-conducted numerical solutions.

The flow variables are defined on the collocated arrangement, and the spatial discretisation is by second order central finite difference. The convective and viscous terms are time-updated by the 4th-order RungeKutta method. A fractional step method is employed for coupling the velocity and pressure fields.

Note that, although the membrane is fixed in space, time-updated position of membrane is introduced in the following to constitute a general closed system. 


\section{A.2.1 Discrete-forcing immersed boundary method: consistent direct discretisation approach}

Immersed boundary method is probably the most widely employed method on a fixed mesh non-conforming to the object surface, among which discrete-forcing (DF) immersed boundary (IB) approach offers a sharp interface treatment by incorporating the boundary conditions of interfacial velocity into the discretised governing equations.

In the early developing stage of the DF approach, discretisation of the pressure Poisson equation was performed uniformly without distinguishing the computational cell containing the interface (boundary cell). Ikeno and Kajishima [29] pointed out the importance of considering the distance from the interface to the adjacent-cell centre for discretising the pressure Poisson equation to strictly satisfy the mass conservation at the boundary cell. The idea is evolved into the concept of "consistent discretisation" of the pressure equation with that of the N-S equation or "consistent coupling" of the incompressible velocity and pressure fields. Later, Sato et al. [27] and Takeuchi et al. [28] developed a method that directly discretises the N-S equation even at the grid points adjacent to the interface, while, at the same time, ensuring the consistency between the incompressible velocity and pressure fields. By their "consistent direct discretisation" for the DF-IB approach, the non-slip condition on the interface was strictly imposed in a discrete sense while satisfying the mass and momentum conservations, which enable capturing the sharp distribution of the velocity and pressure at the interface. This idea makes a clear contrast with the strategy of the early DF-IB approach (including Refs.[29, 30, 31]) that completes the time integration of the velocity at the boundary cell by just assigning an interpolated value (without solving the momentum conservation in the vicinity). Three-dimensional implementation of the consistent direct discretisation is straightforward, and the method was verified for trajectory of a particle travelling in 3-D Taylor-Green vortices [28] and applied to a flow through a woven metal structure [32].

In this study, we propose a new DF formulation (based on the consistent direct discretisation) with permeation by incorporating a volumetric flux driven by discontinuous pressure across membrane, and in this section the validity of the proposed method is established through comparisons with static pressure values for no-permeable case and an analytical prediction of permeation across a membrane placed in a 2-D parallel channel.

In the following, the consistent direct discretisation (with non-permeable interface) is briefly reviewed to facilitate the discretisation study of permeated pressure equation in $\S$ A.2.2. The details of the following method for the non-permeable interface are found in Refs. [27, 28]. 


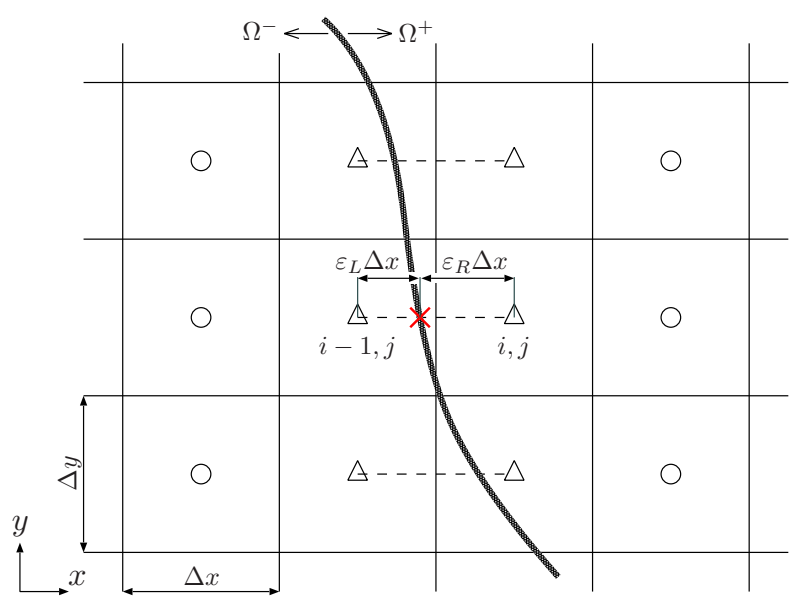

Figure A1: Schematic of the membrane (immersed object) on a fixed Cartesian mesh system. The boundary cells are labelled by triangular symbol, and other fluid cells are by circular symbol. The grid lines of the boundary cells $(i-1, j)-(i, j)$ are separated by a permeable membrane. The intersecting point of the membrane with the grid line connecting the centres of the boundary cells is represented by " $\times$ " symbol.

Fig. A1 shows a schematic of a membrane in an incompressible fluid in two dimensions. Hereafter, the computational cells partitioned by the object boundary are referred to as "boundary cells", as represented by triangular symbol in the figure, which are typically characterised by the segmented centre-to-centre lines (connecting the adjacent cell centres shown by the broken lines) by the membrane. For the boundary cell $(i, j)$ in Fig. A1, the discretisations incorporating the boundary conditions are explained for the following equations and procedure in two dimensions: the N-S equation in the $x$ direction, the pressure equation and the velocity correction (based on a fractional step method).

In the following, $\overline{(\cdot)}$ represents an interpolation operator of second-order accuracy, and $\delta_{x_{k}}$ is an operator of second-order central finite difference in the $x_{k}$ direction. The velocities at the cell face are denoted by $U_{k}(k=1,2)$ or $(U, V)$, and the fractional-step velocities (by excluding the pressure gradient) at the cell centre and cell face are represented by $u_{k}^{* *}$ and $U_{k}^{* *}$, respectively. The subscript "b" stands for a value on the membrane surface, and $\phi_{k}(k=2, \cdots, 5)$ are interpolation/extrapolation functions applied on the boundary cells (given later).

- Convective term (N-S equation)

$$
\begin{aligned}
{\left[\frac{\partial\left(U_{k} u\right)}{\partial x_{k}}\right]_{i, j}=} & \frac{1}{\Delta x}\left\{U_{i+\frac{1}{2}, j}\left(\bar{u}^{x}\right)_{i+\frac{1}{2}, j}-U_{i-\frac{1}{2}, j}\left[\phi_{3}(u)\right]_{i-\frac{1}{2}, j}\right\} \\
& +\frac{1}{\Delta y}\left\{V_{i, j+\frac{1}{2}}\left(\bar{u}^{y}\right)_{i, j+\frac{1}{2}}-V_{i, j-\frac{1}{2}}\left(\bar{u}^{y}\right)_{i, j-\frac{1}{2}}\right\}
\end{aligned}
$$


- Viscous term (N-S equation)

$$
\frac{1}{\operatorname{Re}}\left[\frac{\partial^{2} u}{\partial x_{j}^{2}}\right]_{i, j}=\frac{1}{\operatorname{Re}}\left[\frac{1}{0.5\left(\varepsilon_{R}+1\right) \Delta x}\left\{\left(\delta_{x} u\right)_{i+\frac{1}{2}, j}-\left(\frac{u_{i, j}-u_{\mathrm{b}}}{\varepsilon_{R} \Delta x}\right)\right\}+\left(\delta_{y} \delta_{y} u\right)_{i, j}\right]
$$

- Pressure Poisson equation

$$
\begin{aligned}
& \frac{\Delta t}{\Delta x}\left\{\left(\delta_{x} p^{n+1}\right)_{i+\frac{1}{2}, j}-\left[\phi_{5}\left(\frac{\delta p^{n+1}}{\delta x}\right)\right]_{i-\frac{1}{2}, j}\right\}+\Delta t\left(\delta_{y} \delta_{y} p^{n+1}\right)_{i, j} \\
= & \frac{1}{\Delta x}\left(U_{i+\frac{1}{2}, j}^{* *}-\left[\phi_{2}\left(U^{* *}\right)\right]_{i-\frac{1}{2}, j}\right)+\left(\delta_{y} V^{* *}\right)_{i, j},
\end{aligned}
$$

- Velocity correction procedure

$$
\begin{aligned}
& u_{i, j}^{n+1}=u_{i, j}^{* *}-\Delta t\left[\phi_{4}\left(\frac{\delta p^{n+1}}{\delta x}\right)\right]_{i, j} \\
& U_{i-\frac{1}{2}, j}^{n+1}=U_{i-\frac{1}{2}, j}^{* *}-\Delta t\left[\phi_{5}\left(\frac{\delta p^{n+1}}{\delta x}\right)\right]_{i-\frac{1}{2}, j}
\end{aligned}
$$

where

$$
\begin{aligned}
& {\left[\phi_{2}\left(U^{* *}\right)\right]_{i-\frac{1}{2}, j}=\frac{1}{\varepsilon_{R}+0.5}\left\{\left(\varepsilon_{R}-0.5\right) U_{j+\frac{1}{2}}^{* *}+u_{\mathrm{b}}\right\}} \\
& {\left[\phi_{3}(u)\right]_{i-\frac{1}{2}, j}=\frac{1}{\varepsilon_{R}}\left\{\left(\varepsilon_{R}-0.5\right) u_{i, j}+0.5 u_{\mathrm{b}}\right\},} \\
& {\left[\phi_{4}\left(\frac{\delta p}{\delta x}\right)\right]_{i, j}=\frac{1}{\varepsilon_{R}+0.5}\left\{\varepsilon_{R}\left(\delta_{x} p\right)_{i+\frac{1}{2}, j}+0.5\left(\frac{\delta p}{\delta x}\right)_{\mathrm{b}}\right\},} \\
& {\left[\phi_{5}\left(\frac{\delta p}{\delta x}\right)\right]_{i-\frac{1}{2}, j}=\frac{\varepsilon_{R}-0.5}{\varepsilon_{R}+0.5}\left(\delta_{x} p\right)_{i+\frac{1}{2}, j} .}
\end{aligned}
$$

and $\varepsilon_{L}$ and $\varepsilon_{R}$ are as shown in Fig. A1. The superscript $n+1$ stands for the next time level.

As described above, by considering the distance to the object surface for discretising the equations, the momentum conservation is satisfied even in the boundary cell in a discrete sense. This is different in philosophy from the early DF-IB approaches which assign the velocity near the interface by interpolation to satisfy the no-slip at the interface. For this reason, the above discretisation procedure claims the "direct discretisation" even in the immediate vicinity of the interface. Note that, while the primary variables on the cell centres are solved by direct discretisation, the secondary variables (such as cell-face velocities and gradients) are obtained by interpolation and extrapolation. Further, the correction procedure (Eq.(A7), using the pressure at the next time level obtained by solving the pressure Poisson equation, Eq.(A6)) determines the velocity in the boundary cell to satisfy the non-slip condition at the interface, and therefore, the mass 
conservation is guaranteed simultaneously in the discrete sense. This procedure guarantees the consistency between the incompressible velocity and pressure fields.

In a boundary cell of $\varepsilon_{R} \simeq 0$, the terms with $\varepsilon_{R}^{-1}$ in Eqs. (A4) and (A5) may cause numerical instability. In order to avoid this, we introduce a threshold $\varepsilon_{\text {limit }}$ for evaluating the convective and viscous terms, and the discretisation stencil is expanded upon appearance of those cells $\left(\varepsilon_{R}<\varepsilon_{\text {limit }}\right)$. The detail of this treatment is found in Ref. [28].

In the above, the discretisations are presented only for the 2-D region in the right-hand side of the interface in Fig. A1. For the flow around a thin object (i.e., non-permeable membrane, here), the similar discretisation is applied for the region in the left-hand side of the interface [28]. For the stationary membrane, as assumed in the present paper, $u_{\mathrm{b}}=0$ is prescribed in the above discretisations, while, for a moving boundary problem, the fluid velocity on the membrane $u_{\mathrm{b}}$ needs to be specified to coincide with the membrane velocity (viz. Lagrangian variable) due to the no-slip condition. As a general procedure in our study, two neighbouring Lagrangian markers (consisting of the membrane) closest to the " $\times$ " point in Fig. A1 are identified, and a linear interpolation of those marker velocities finally determines the fluid boundary velocity $u_{\mathrm{b}}$.

\section{A.2.2 Pressure Poisson equation with permeation}

As Miyauchi et al. [26] pointed out, in order to reproduce the permeation of incompressible fluid through a permeable membrane on a fixed fluid mesh system (non-conforming to the membrane surface, in general), sharp representation of the membrane, particularly capturing the discontinuity of the pressure across the membrane, is of critical importance. For this reason, the above consistent direct discretisation for DF-IB method is a suitable approach, as the interfacial pressure distribution is consistent with the no-slip condition (and more generally, the pressure reflects the local mass conservation for an incompressible fluid). In this subsection, the above discretisation strategy is extended for permeable interface, where the no-slip condition on the interface needs to be relaxed to allow the surface-normal permeate flux.

In the following, permeate flux is incorporated into the above DF formulation. The specific change appears as a new pressure equation, by which the consistency between the incompressible velocity and pressure fields is extended for permeable interface.

For the non-permeable interface ( $\S$ A.2.1), pressure equations can be constructed on both sides of the interface independently, whereas the permeable interface relates the pressures in both sides through Eq.(A3). 
When the membrane is placed between the cells $(i-1, j)$ and $(i, j)$, as shown in Fig. A1, the pressure equations on those boundary cells are subjected to the following modifications (based on the equation of continuity in the boundary cell):

- Pressure equation in the boundary cell $(i-1, j)$

$$
\begin{aligned}
& \frac{\Delta t}{\Delta x}\left\{\left[\phi_{5}^{L}\left(\frac{\delta p^{n+1}}{\delta x}\right)\right]_{i-\frac{1}{2}, j}-\left(\delta_{x} p^{n+1}\right)_{i-\frac{3}{2}, j}\right\}+\Delta t\left(\delta_{y} \delta_{y} p^{n+1}\right)_{i-1, j} \\
& =\frac{1}{\Delta x}\left(\left[\phi_{2}^{L}\left(U^{* *}\right)\right]_{i-\frac{1}{2}, j}-U_{i-\frac{3}{2}, j}^{* *}\right)+\left(\delta_{y} V^{* *}\right)_{i-1, j}
\end{aligned}
$$

- Pressure equation in the boundary cell $(i, j)$

$$
\begin{aligned}
& \frac{\Delta t}{\Delta x}\left\{\left(\delta_{x} p^{n+1}\right)_{i+\frac{1}{2}, j}-\left[\phi_{5}^{R}\left(\frac{\delta p^{n+1}}{\delta x}\right)\right]_{i-\frac{1}{2}, j}\right\}+\Delta t\left(\delta_{y} \delta_{y} p^{n+1}\right)_{i, j} \\
& =\frac{1}{\Delta x}\left(U_{i+\frac{1}{2}, j}^{* *}-\left[\phi_{2}^{R}\left(U^{* *}\right)\right]_{i-\frac{1}{2}, j}\right)+\left(\delta_{y} V^{* *}\right)_{i, j} .
\end{aligned}
$$

Here, the interpolation functions $\left[\phi_{2}^{\{L, R\}}\left(U^{* *}\right)\right]_{i-\frac{1}{2}, j}$ and $\left[\phi_{5}^{\{L, R\}}(\partial p / \partial x)\right]_{i-\frac{1}{2}, j}$ are identified with the surface pressures and the gradients as follows:

$$
\begin{gathered}
{\left[\phi_{2}^{L}\left(U^{* *}\right)\right]_{i-\frac{1}{2}, j}=\frac{1}{\varepsilon_{L}+0.5}\left\{\left(\varepsilon_{L}-0.5\right) U_{i-\frac{3}{2}, j}^{* *}+u_{\mathrm{b}-0}^{* *}\right\}} \\
{\left[\phi_{2}^{R}\left(U^{* *}\right)\right]_{i-\frac{1}{2}, j}=\frac{1}{\varepsilon_{R}+0.5}\left\{\left(\varepsilon_{R}-0.5\right) U_{i+\frac{1}{2}, j}^{* *}+u_{\mathrm{b}+0}^{* *}\right\}} \\
{\left[\phi_{5}^{L}\left(\frac{\delta p}{\delta x}\right)\right]_{i-\frac{1}{2}, j}=\frac{1}{\varepsilon_{L}+0.5}\left\{\left(\varepsilon_{L}-0.5\right)\left(\delta_{x} p^{n+1}\right)_{i-\frac{3}{2}, j}+\left(\frac{\delta p}{\delta x}\right)_{\mathrm{b}-0}\right\}} \\
{\left[\phi_{5}^{R}\left(\frac{\delta p}{\delta x}\right)\right]_{i-\frac{1}{2}, j}=\frac{1}{\varepsilon_{R}+0.5}\left\{\left(\varepsilon_{R}-0.5\right)\left(\delta_{x} p^{n+1}\right)_{i+\frac{1}{2}, j}+\left(\frac{\delta p}{\delta x}\right)_{\mathrm{b}+0}\right\}}
\end{gathered}
$$

where the subscripts $b \mp 0$ stand for the limiting values in the left- and right-hand sides of the interface.

Note that, for the no-permeable case ( $\S$ A.2.1), the fluid velocity on the surface, $u_{\mathrm{b}}$, is the same as the membrane velocity. Instead, in the above equations, the intermediate fluid velocities on the membrane surface $u_{\mathrm{b} \pm 0}^{* *}$ are introduced tentatively. Assuming that $u_{\mathrm{b} \pm 0}^{* *}$ are also time-updated (like Eq.(A7a)) using the pressure gradient $(\partial p / \partial x)_{\mathrm{b} \pm 0}$, we obtain the following equation by enforcing Eq. (A3) with the next-step velocity $u_{\mathrm{b} \pm 0}^{n+1}$ :

$$
\begin{aligned}
u_{\mathrm{b} \pm 0}^{* *}-\Delta t\left(\frac{\delta p^{n+1}}{\delta x}\right)_{\mathrm{b} \pm 0} & =u_{\mathrm{b} \pm 0}^{n+1} \\
& =u_{\mathrm{m}}^{n+1}+\operatorname{Re} L n_{x}^{n+1}[p]^{n+1}
\end{aligned}
$$




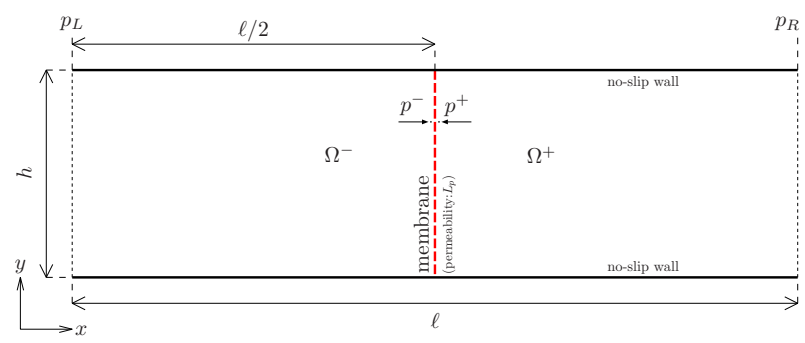

(a) Perpendicular to the walls

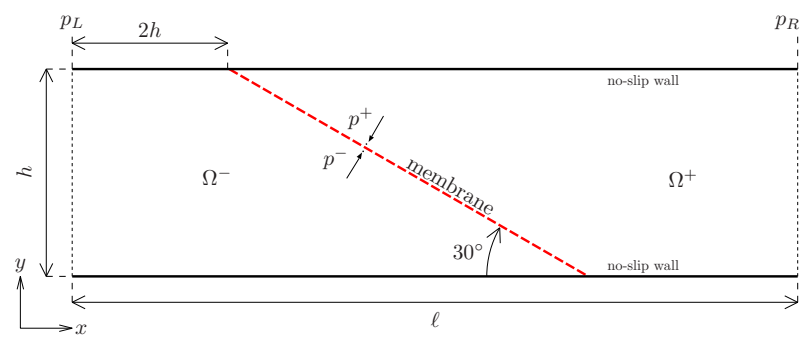

(b) Oblique to the walls

Figure A2: Schematics of a straight fixed membrane in a 2-D channel. (a) Perpendicular to the top and bottom walls. (b) Oblique to the channel walls. Aspect ratio of the domain is fixed to $\ell / h=5$ for both cases.

where $u_{\mathrm{m}}$ is the translating velocity of membrane and $n_{x}$ is the $x$ component of $\boldsymbol{n}$. By substituting the interpolation functions (Eqs. (A14) and (A15)) and Eq.(A16) into the pressure equations (A12) and (A13), the pressure fields in association with a permeate flux should satisfy the following equations in the respective sides of the membrane:

- Permeated pressure equation at the boundary cell $(i-1, j)$

$$
\begin{aligned}
& -\frac{\Delta t}{\left(\varepsilon_{L}+0.5\right) \Delta x}\left\{\left(\delta_{x} p^{n+1}\right)_{i-\frac{3}{2}, j}+\frac{\left.\operatorname{Re} L n_{x}^{n+1} \llbracket p^{n+1}\right]}{\Delta t}\right\}+\Delta t\left(\delta_{y} \delta_{y} p^{n+1}\right)_{i-1, j} \\
& =\frac{u_{\mathrm{m}}^{n+1}-U_{i-\frac{3}{2}, j}^{* *}}{\left(\varepsilon_{L}+0.5\right) \Delta x}+\left(\delta_{y} V^{* *}\right)_{i-1, j}
\end{aligned}
$$

- Permeated pressure equation at the boundary cell $(i, j)$

$$
\begin{aligned}
& \frac{\Delta t}{\left(\varepsilon_{R}+0.5\right) \Delta x}\left\{\left(\delta_{x} p^{n+1}\right)_{i+\frac{1}{2}, j}+\frac{\operatorname{Re} L n_{x}^{n+1} \llbracket p^{n+1} \rrbracket}{\Delta t}\right\}+\Delta t\left(\delta_{y} \delta_{y} p^{n+1}\right)_{i, j} \\
& =\frac{U_{i+\frac{1}{2}, j}^{* *}-u_{\mathrm{m}}^{n+1}}{\left(\varepsilon_{R}+0.5\right) \Delta x}+\left(\delta_{y} V^{* *}\right)_{i, j} .
\end{aligned}
$$

In the implementation, for the closure of the discretised equations, the pressure jump is written down with the difference of the two surface pressures separately obtained by 1st-order extrapolations from the regions away from the interface in both sides of the membrane.

\section{A.3 Validation: flow in 2-D channel with a membrane}

A 2-D parallel channel subjected to a constant pressure difference is set up for validation of the numerical method in $\S$ A.2.2. Figure A2 shows schematics of the computational domain and boundary condition. The longitudinal $(x)$ and transverse $(y)$ sizes of the domain are $\ell$ and $h$, respectively. The top and bottom 


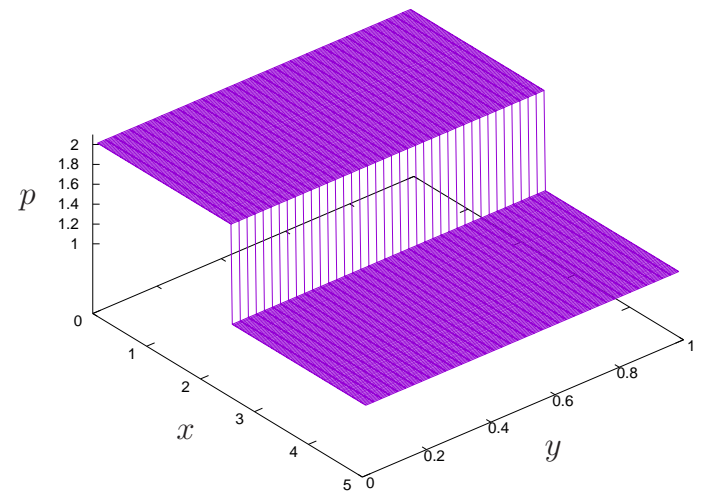

(a) Perpendicular to the walls

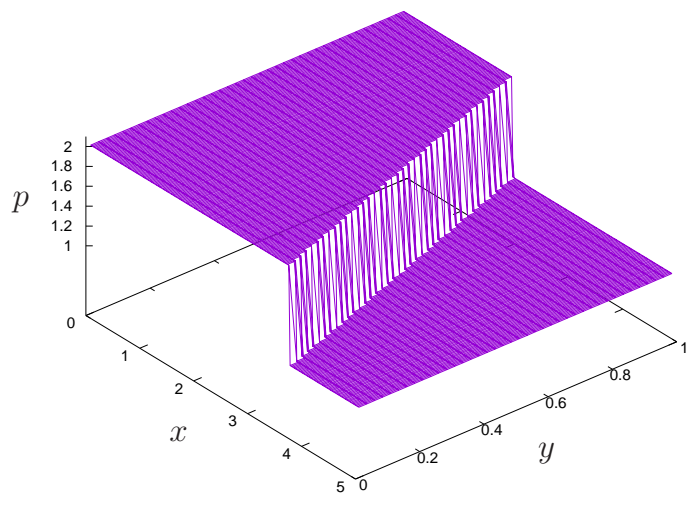

(b) Oblique to the walls

Figure A3: Steady pressure distributions of no-permeable cases for two different membrane configurations; (a) perpendicular and (b) oblique to the walls, as schematically shown in Fig. A2.

boundaries are stationary walls. In the following, we take the membrane length $h$ as the reference length, and the aspect ratio of the domain is fixed to $\ell / h=5$. The reference velocity is taken as the maximum velocity, $U_{\max }$, in steady state of no-membrane case $(L \rightarrow \infty)$ in the 2-D channel. The pressures on the left and right boundaries are fixed to be $p_{L}=2$ and $p_{R}=1$, respectively, and the flow field driven by the external pressure difference $\Delta p\left(=p_{L}-p_{R}\right)$ through the membrane is studied. Two cases of membrane configuration are tested to compare the effect of membrane alignment to the grid line: Fig. A2(a) shows a straight membrane perpendicular to the top and bottom walls with the alignment on the cell face along the $y$ axis, and Fig. A2(b) is straight membrane oblique to the walls at the angle of 30 [deg] with respect to the $-x$ axis. No fluid variables are defined on the membranes. Hereafter, the Reynolds number is set to $\operatorname{Re}=1$, and unless specified otherwise, the grid spacing $\Delta$ is uniform in both directions with the spatial resolution $h / \Delta=40$.

As a first step of validation, a no-permeable case $(L=0)$ is solved. Under this condition, stationary solution is obtained with the steady pressures in the respective regions (i.e., the left- and right-hand sides of the membrane) matching with the boundary pressure values. Fig. A3(a) shows the numerical result of the pressure distribution. From the figure, constant pressure distributions are obtained in both sides of the membrane with the pressure discontinuity $\llbracket p \rrbracket=1$ at the membrane position. The pressure jump is captured over one cell. The orders of magnitude of the $L_{2}$ and $L_{\infty}$ errors in the pressure from the boundary pressures are $10^{-9}$ and $10^{-11}$, respectively.

For the oblique configuration of membrane (Fig.A3(b)), under a unit external pressure difference along the longitudinal distance $\ell$, the steady pressure distributions are also obtained in both sides of the membrane 


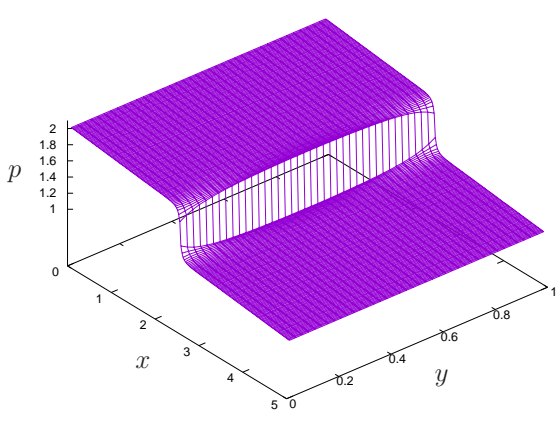

(a) $L=0.1$

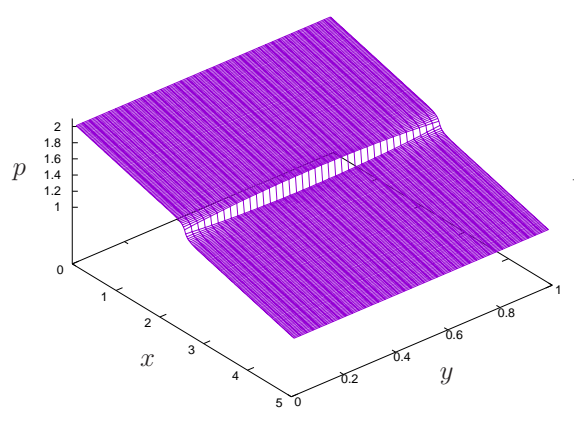

(b) $L=1$

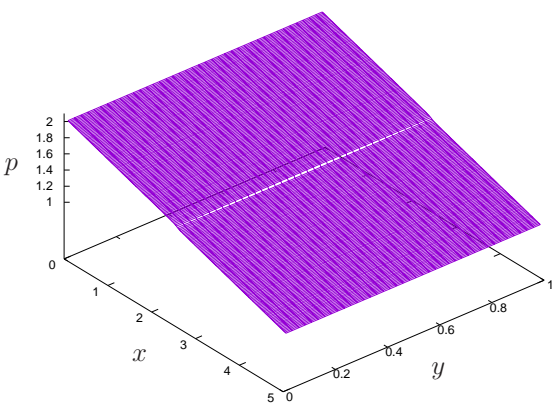

(c) $L=10$

Figure A4: Steady pressure distributions for three different permeabilities, $L=0.1,1$ and 10 (correspondingly, $M=0.5,5$ and 50 ).

with a sharp pressure difference, as shown in Fig. A3(b). The orders of magnitude of the $L_{2}$ and $L_{\infty}$ errors in the pressure from the boundary pressures are found to be $10^{-9}$ and $10^{-11}$ for the following spatial resolutions: $h / \Delta x=10,20$ and 40 .

For non-zero permeability case with the grid-aligned configuration of Fig. A2(a), Figs. A4(a) A4(c) plot the pressure distributions for the following three permeabilities: $L=0.1,1$ and 10 . The pressure gaps along the membrane (i.e., in the $y$ direction) are no longer constant. Due to the permeate flux across membrane, steady pressure gradients in both sides of the membrane are approximately constant. The pressure distribution of Fig. A4(c) shows that the membrane of $L=10$ is highly leaky.

Figures A3 and A4 show that the permeate flux model is reasonably implemented in the context of incompressible flow field including the limiting case of non-permeable membrane.

Flow rate of permeation flow in a 2-D parallel channel is discussed as a function of permeability. In the channel of Fig. A2(a), the flow rate is denoted by $Q$, and normalised by the flow rate of the 2-D Poiseuille flow (i.e., $L \rightarrow \infty), Q_{\mathrm{P}}$, under the same prescribed pressure gradient. Figure A5 plots the numerical result of $Q / Q_{\mathrm{P}}$ against the non-dimensional permeability $M=L \ell / h$, where $M$ is varied in the following range: $M=5 \times 10^{-3}, 5 \times 10^{-2}, 5 \times 10^{-1}, 5,50$ and 500 (correspondingly, $L=10^{-3}, 10^{-2}, 10^{-1}, 1,10$ and $10^{2}$ ). Also plotted is an independently conducted numerical result by a monolithic strong coupling of the flow field and permeate flux [26], and the present numerical result shows good agreement. Miyauchi et al. [26] further showed that the ratio of the 2-D flow rates, $Q / Q_{\mathrm{P}}$, can be modelled by the following equation:

$$
\frac{Q}{Q_{\mathrm{P}}}=-\frac{96 M^{2}}{\sqrt{1+8 M}} \operatorname{arctanh}\left[\frac{1}{\sqrt{1+8 M}}\right]+12 M .
$$

Figure A5 also shows a comparison with the above equation, and the numerical results by the two different numerical methods fall on the above model. 


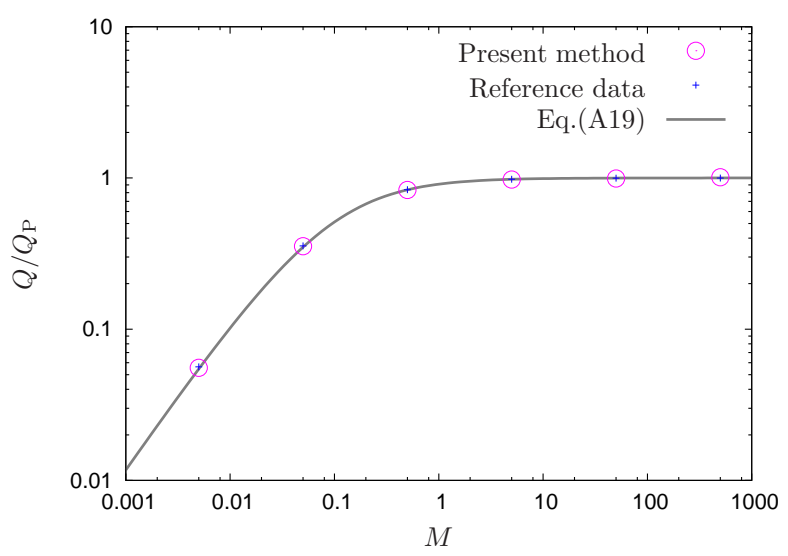

Figure A5: Normalised flow rate $Q / Q_{\mathrm{P}}$ as a function of the non-dimensional permeability $M$. The circular symbol is the numerical result by the present method and the line represents the prediction by an analytical model, Eq.(A19). For comparison, reference numerical data by the method in Ref. [26] (with no deflection of membrane) are plotted. The reference data are obtained for the same configuration and the boundary conditions as Fig. A2(a) with spatial resolutions of $h / \Delta=50$ and 24 Lagrangian markers along the membrane (length: $h$ ), which exhibits negligible difference $(<0.2 \%$ in maximum) in flow rates under a low resolution case of 32 fluid cells and 18 Lagrange markers along $h$.

The above results suggest the validity of the consistent direct discretisation incorporating the pressure discontinuity associated with permeate flux through a fixed membrane.

\section{Acknowledgements}

This work is partly supported by Grant-in-Aid (B) No. 17H03174 of the Japan Society for the Promotion of Science (JSPS). One of the authors, S.M., gratefully acknowledges the Leading Young Researcher Overseas Visit Program of Tohoku University, Japan.

\section{REFERENCES}

[1] J. Lin, W. Ye, H. Zeng, H. Yang, J. Shen, S. Darvishmanesh, P. Luis, A. Sotto and B. Van der Bruggen, "Fractionation of direct dyes and salts in aqueous solution using loose nanofiltration membranes", Journal of Membrane Science, Vol.477, pp.183-193 (2015)

[2] S. Loeb and S. Sourirajan, "Sea Water Demineralization by Means of an Osmotic Membrane", Advances in Chemistry, Vol.38, pp.117-132 (1963)

[3] S. Manjikian, "Desalination Membranes from Organic Casting Solutions", Industrial \& Engineering Chemistry Product Research and Development, Vol.6 (1), pp.23-32 (1967)

[4] X. Gong, Z. Gong and H. Huang, "An immersed boundary method for mass transfer across permeable moving interfaces", Journal of Computational Physics, 278, pp.148-168 (2014)

[5] R.K. Jain, “An indirect way to tame cancer", Scientific American, Vol.310 (2), pp.46-53 (2014)

[6] G.L. Amidon, J. Kou, R.L. Elliott and E.N. Lightfoot, "Analysis of models for determining intestinal wall permeabilities”, Journal of Pharmaceutical Sciences, Vol.69 (12), pp.1369-1373 (1980) 
[7] A.E. Taylor, A.C. Guyton and V.S. Bishop "Permeability of the Alveolar Membrane to Solutes", Circulation Research, Vol.16, pp.353-362 (1965)

[8] J.S. Patton, "Mechanisms of macromolecular absorption by the lungs", Advanced Drug Delivery Reviews, Vol.19, Issue 1, pp.3-36. (1996)

[9] J.S. Patton, J. Bukar and S. Nagarajan, "Inhaled insulin", Advanced Drug Delivery Reviews, Vol.35, Issue 2-3, pp.235-247 (1999)

[10] M. Smola, T. Vandamme and A. Sokolowski, "Nanocarriers as pulmonary drug delivery systems to treat and to diagnose respiratory and non respiratory diseases", International Journal of Nanomedicine, Vol.3 (1): pp.1-19 (2008)

[11] K.-G. Zhou, K. S. Vasu, C. T. Cherian, M. Neek-Amal, J. C. Zhang, H. Ghorbanfekr-Kalashami, K. Huang, O. P. Marshall, V. G. Kravets, J. Abraham, Y. Su, A. N. Grigorenko, A. Pratt, A. K. Geim, F. M. Peeters, K. S. Novoselov and R. R. Nair, "Electrically controlled water permeation through graphene oxide membranes", Nature, Vol.559, pp.236-240, (2018)

[12] J.G. Wijmans, S. Nakao and C.A. Smolders, "Flux limitation in ultrafiltration: osmotic pressure model and gel layer model”, Journal of Membrane Science, Vol.20, pp.115-124 (1984)

[13] H. Yoshida and L. Bocquet, "Labyrinthine water flow across multilayer graphene-based membranes: Molecular dynamics versus continuum predictions", The Journal of Chemical Physics, Vol.144, 234701 (2016)

[14] A. Verniory, R. Du Bois, P. Decoodt, J.P. Gassee and P.P. Lambert, "Measurement of the Permeability of Biological Membranes Application to the glomerular wall", The Journal of General Physiology, Vol.62, pp.489-507 (1973)

[15] A. Chahboun, R. Coratger, F. Ajustron, J. Beauvillain, P. Aimar and V. Sanchez, "First investigations on the use of scanning tunnelling microscopy (STM) for the characterisation of porous membranes", Journal of Membrane Science, Vol.67, pp.295-300 (1992)

[16] J.I. Calvo, A. Hernández, G. Caruana and L. Martínez, "Pore Size Distributions in Microporous Membranes: I. Surface Study of Track-Etched Filters by Image Analysis", Journal of Colloid and Interface Science, Vol.175, Issue 1, pp.138-150 (1995)

[17] E. Honold and E.L. Skau, "Application of Mercury-Intrusion Method for Determination of Pore-Size Distribution to Membrane Filters", Science, Vol.120 Issue 3124, pp.805-806 (1954)

[18] A.A. Liabastre and C. Orr, "An evaluation of pore structure by mercury penetration", Journal of Colloid and Interface Science, Vol.64, Issue 1, pp.1-18 (1978)

[19] L. Palacio, P. Praádanos, J.I. Calvo and A. Hernaández, "Porosity measurements by a gas penetration method and other techniques applied to membrane characterization", Thin Solid Films, Vol.348, pp.22-29 (1999)

[20] "Improved accuracy in bubble point measurement", Filtration + Separation, Vol.54, Issue 4, pp.38-41 (2017) DOI : $10.1016 /$ S0015-1882(18) 30046-6

[21] M. Brun, A. Lallemand, J.F. Quinson and C. Eyraud, "A new method for the simultaneous determination of the size and shape of pores: the thermoporometry", Thermochimica Acta, Vol.21, pp.59-88 (1977)

[22] S. Nakao and S. Kimura, "Models of membrane transport phenomena and their applications for ultrafiltration data" Journal of Chemical Engineering of Japan, Vol.15 (3), pp.200-205 (1982)

[23] J. Happel and H. Brenner, Low Reynolds Number Hydrodynamics, Prentice-Hall (1965) 
[24] H. Padé , "Sur la représentation approchée d'une fonction par des fractions rationnelles", Annales Scientifiques de l'École Normale Supérieure, Série 3, Vol.9, pp.3-93 (1892)

[25] A. Baker, Jr., Essentials of Padé Approximants, Academic Press, New York (1975)

[26] S. Miyauchi, S. Takeuchi and T. Kajishima, "A numerical method for interaction problems between fluid and membranes with arbitrary permeability for fluid", Journal of Computational Physics Vol.345, pp.33-57 (2017) http://doi.org/10.1016/j.jcp.2017.05.006

[27] N. Sato, S. Takeuchi, T. Kajishima, M. Inagaki and N. Horinouchi, "A consistent direct discretization scheme on Cartesian grids for convective and conjugate heat transfer", Journal of Computational Physics Vol.321, pp.76104 (2016) http://doi.org/10.1016/j.jcp.2016.05.034

[28] S. Takeuchi, H. Fukuoka, J. Gu and T. Kajishima, "Interaction problem between fluid and membrane by a consistent direct discretisation approach", Journal of Computational Physics, Vol.371, pp.1018-1042 (2018) http://doi.org/10.1016/j.jcp.2018.05.033

[29] T. Ikeno and T. Kajishima, "Finite-difference immersed boundary method consistent with wall conditions for incompressible turbulent flow simulations", Journal of Computational Physics, Vol.226, pp.1485-1508 (2007)

[30] J. Mohd-Yusof, "Combined immersed-boundary/B-spline methods for simulations of flow in complex geometries", Annual Research Briefs, Center for Turbulence Research, Stanford University, pp.317-327 (1997)

[31] E.A. Fadlun, R. Verzicco, P. Orlandi and J. Mohd-Yusof, "Combined immersed-boundary finite-difference methods for three-dimensional complex flow simulations", Journal of Computational Physics, Vol.161, pp.35-60 (2000)

[32] N. Sato, S. Takeuchi, T. Kajishima, M. Inagaki and N. Horinouchi, "Accuracy improvement of the Cartesian grid method for numerical simulations of heat transfer and fluid flow", JSME Fluids Engineering Devision Newsletter, Vol.2016.02

http://www.jsme-fed.org/papertech/2016_01/002.html 